\title{
AIR ENTRAINMENT AND FREE SURFACE MODELING OF FULLY TURBULENT FLOW NEAR THE BROAD-CRESTED WEIRS
}

\author{
${ }^{1}$ Martin ORFÁNUS ${ }^{*},{ }^{2}$ Ján RUMANN \\ ${ }^{1,2}$ Faculty of Civil Engineering, Slovak University of Technology in Bratislava \\ Radlinského 11, 81005 Bratislava, Slovakia \\ e-mail: ${ }^{1}$ martin.orfanus@stuba.sk, ${ }^{2}$ jan.rumann@stuba.sk
}

Received 2 January 2018; accepted 23 March 2019

\begin{abstract}
The research was carried out at the Department of Hydraulic Engineering, Faculty of Civil Engineering, Slovak University of Technology in Bratislava with close cooperation with Institute of Water Structures, Faculty of Civil Engineering, Brno University of Technology. One of the important goals was the determination of the most accurate approach related to the computational fluid dynamics modeling of the air-water interaction. Research consists of two main research methods to ensure the accuracy, set up the possibilities of cooperation and the results control. The first method was the physical model of broad-crested weir installed in collapsible canal in hydraulic laboratory and the second was numerical computational fluid dynamics model of broad-crested weir created in same scale. In free surface flows the air entrainment phenomena plays an important role. Air entrainment is affecting the volume fraction, velocity field, energy dissipation and many other parameters related to dynamic behavior of water near the crests. Different approaches were carried out to identify most accurate computational fluid dynamics setup compared to physical model measurements. Compared results and the accuracy assessment are summarized and the best computational fluid dynamics parameterization is recommended.
\end{abstract}

Keywords: Broad-crested weir, Physical model, computational fluid dynamics, Hybrid modeling, Hydraulic research

\section{Introduction}

The broad crested weirs are frequently used to determine the discharge. The broad crested weir crest is straight; the upstream and downstream walls are vertical and the

\footnotetext{
${ }^{*}$ Corresponding Author
} 
discharge coefficient is unvarying in moderately high rectangular sharp-edged weirs with a broad crest and without tangential contraction $0.1 \leq h / t \leq 0.3$ and $h / s<0.15$, where $s[\mathrm{~m}]$ is the height of the weir [1]. Standard criteria in terms if the weir is sharp or broad-crested is $t>2 / 3 h$ and one of most general empirical estimation of discharge above the broad-crested weir is

$$
Q=\frac{2}{3} \mu_{P} b \sqrt{2 g h_{0}^{2 / 3}}
$$

where $Q$ is the discharge; $b$ is the width; $\mu_{P}$ is the discharge coefficient and $h_{0}$ is the upstream total head according to Fig 1.

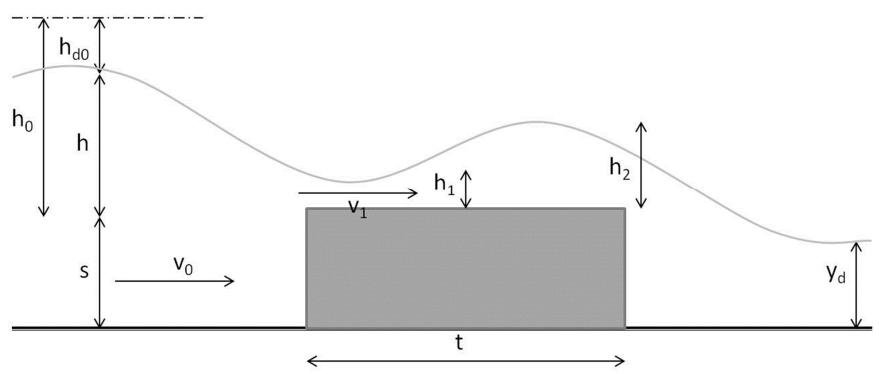

Fig. 1. Broad-crested weir scheme, on the basis of [2]

The exactness of the simulated results was confirmed on the physical model experiments of the current case (Fig. 2) as well as other cases of free surface or pressurized water flow [3].

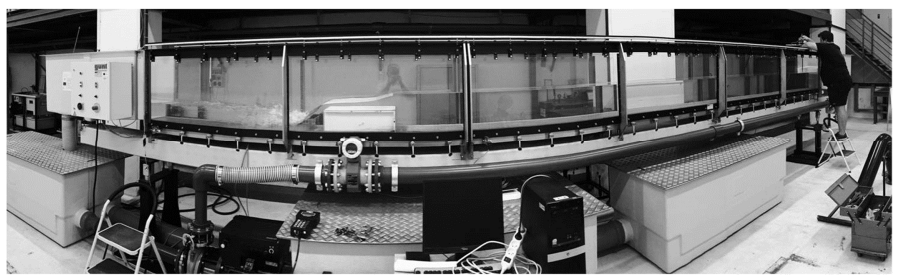

Fig. 2. Broad-crested weir - physical model

The physical model took a part of research as the main calibration and verification researching method with measurement of the water levels in different locations along the canal and velocity fields using a point gauge and an acoustic Doppler 2D anemometer (FlowTracker). The velocity measurements were performed in 5 vertices with a division at a depth of $0.03 \mathrm{~m}$ (Fig. 3) near the object of broad crested weir with dimensions of $t=0.497 \mathrm{~m}$ and $s=0.151 \mathrm{~m}$.

To locate and investigate these phenomena properly by using Computer Fluid Dynamic (CFD) modeling capabilities the fact of air entrainment, turbulence and free surface tracking has to be considered. 

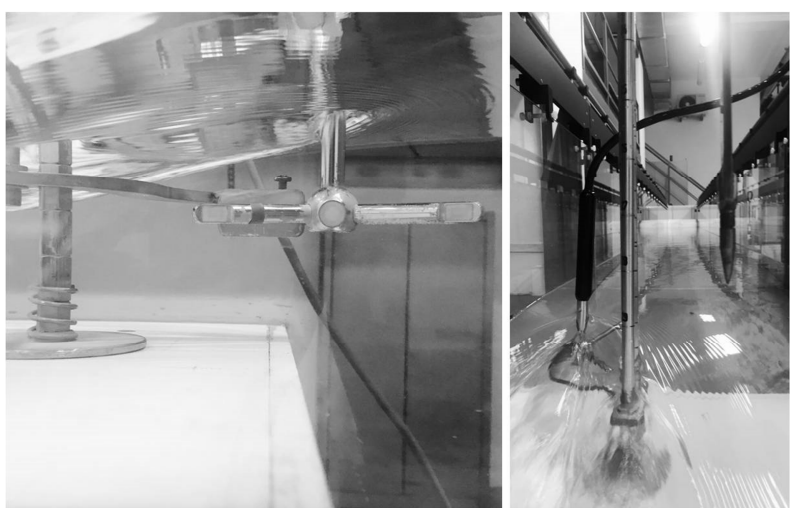

Fig. 3. Broad-crested weir (velocity measurement)

The single-phase model cannot accurately capture the air entrainment of the freesurface vortex because it is a two-phase flow. So, multiphase models have also been studied. The most adopted and reliable method is the Volume of Fluid (VoF) multiphase model to capture the wave characteristics of the free surface, which agreed with experimental observations as well. The VoF method, the formation of vortices and velocity field deformation is included in the FLOW-3D code used to determine the flow behavior near the broad -crested weir, which shows that vortices calculated to have the highest values were consistent with those of air-entraining vortices [4].

Flow with free surface and a turbulent behavior may be so intensive that the distraction of its sharp interface will occur to the point of entraining air into the flow. The free surface flow tracking is based on the algorithms of VoF method. This function represents the volume of fluid \#1 per unit volume and satisfies the equation

$$
\frac{\partial F}{\partial t}+\frac{1}{V_{F}}\left(\frac{\partial F A_{x} u}{\partial x}+R \frac{\partial F A_{y} v}{\partial y}+\frac{\partial F A_{z} w}{\partial z}+\xi \frac{F A_{x} u}{x}\right)=F_{D I F}+F_{S O R}
$$

where

$$
F_{D I F}=\frac{1}{V_{F}}\left[\left(v_{F} A_{x} \frac{\partial F}{\partial x}\right)+R \frac{\partial}{\partial y}\left(v_{F} A_{y} R \frac{\partial F}{\partial y}\right)+\frac{\partial}{\partial z}\left(v_{F} A_{z} R \frac{\partial F}{\partial z}\right)+\xi \frac{v_{F} A_{x} F}{x}\right] .
$$

The diffusion coefficient is defined as $v_{F}=c_{F} \mu / \rho$ where $c_{F}$ is a constant whose reciprocal is sometimes referred to as a turbulent Schmidt number. This diffusion term only makes sense for the turbulent mixing of two fluids whose distribution is defined by the function $F$.

The term time rate of change of the volume fraction of fluid \#1 associated with the mass source for fluid $\# 1,\left(F_{S O R}\right)$ corresponds to the density source $\left(R_{S O R}\right) ; F_{S O R}$ is the time rate of change of the volume fraction of water associated with the mass source for water. $F_{D I F}$ is the fluid diffusion. 
The interpretation of function $F$ depends on the type of problem being solved. Incompressible problems must involve either a single fluid with a free-surface or two fluids and no free-surfaces. For a single fluid, $F$ represents the volume fraction occupied by the fluid. Thus, fluid exists where $F=1$, and void regions correspond to locations where $F=0$. 'Voids' are regions without fluid mass that have a uniform pressure assigned to them. Physically, they represent regions filled with a vapor or gas whose density is insignificant with respect to the fluid density. The phenomena of entraining air can be essential in many applications including hydraulics [5].

Overflow of the water at the broad-crested weirs causes an intensive air entrainment known as hydraulic jump with fast transition from a high-velocity to a low-velocity flow characterized by strong turbulence and air bubble entrainment at the downstream when the overflowing water jets into the receiving pool after passing through a gas phase entrains an quantity of air and creates a submerged two-phase area with a interfacial spot [6].

Air can be entrained into flowing water in a number of ways. Entrainment should develop by the inflow impingement, air dragged by the falling water streams and drops, entrainment by the flow plunging and entrainment by the turbulent outflow. The relative contributions of each of these mechanisms have not been studied in detail [7].

\section{Numerical modeling}

The Reynold's Averaged Navier-Stokes (RANS) conservation equations are derived from the Navier-Stokes equation, by applying the Reynold's decomposition that decomposes the liquid flow properties into their time-mean value and fluctuating component. The mean velocity is defined as a time average for a period that is larger than the time scale of the fluctuations. The time average of the fluctuations over $t$ tends to zero, meaning the turbulence components do not contribute to the bulk mass transport. The time-dependent RANS equations for continuity and momentum conservation are reported below

$$
\begin{aligned}
& \frac{\partial}{\partial x_{i}}\left(\rho \overline{u_{1}}\right)=0 \\
& \rho \frac{\partial \bar{u}_{1}}{\partial t}+\rho \frac{\partial}{\partial x_{j}}\left(\bar{u}_{1} \bar{u}_{j}\right)+\rho \frac{\partial}{\partial x_{j}}\left(\bar{u}_{1}^{\prime} \bar{u}_{i}^{\prime}\right)=-\frac{\partial \bar{p}}{\partial x_{i}}+\mu \frac{\partial^{2} \bar{u}_{i}}{\partial x_{j}^{2}}+\rho g_{i},
\end{aligned}
$$

where $\rho$ is the fluid density; $x_{i}$ is the direction vector; $u_{j}$ is the Reynolds averaged velocity in the $x$ direction; and $g_{i}$ is the sum of body forces. The decomposition of the momentum equation with Reynolds decomposition generates the Reynolds stresses term from the nonlinear convection component. Including turbulence in to the simulations numerical setup is an important part of pre-processing. When vortices are shed from the structure a periodic force is generated, which might lead to the vibration of the structure ore effect the capacity of the structure. Because the Reynolds stresses are unknown 
variables, the realizable $k-\varepsilon$ model proposed by Shih et al. [8] is used to resolve the closure problem.

The realizable $k-\varepsilon$ model suitable for boundary free shear flow applications consists of a turbulent kinetic energy equation and a turbulence energy dissipation rate equation, respectively reported below

$$
\begin{aligned}
& \frac{\partial k}{\partial t}+\bar{u}_{j} \frac{\partial k}{\partial x_{j}}=\frac{\partial}{\partial x_{j}}\left(\frac{v_{t}}{\sigma_{k}} \frac{\partial k}{\partial x_{j}}\right)-\bar{u}_{1} \bar{u}_{j} \frac{\partial \bar{u}_{1}}{\partial x_{j}}-\varepsilon \\
& \frac{\partial \varepsilon}{\partial t}+\bar{u}_{j} \frac{\partial \varepsilon}{\partial x_{i}}=\frac{\partial}{\partial x_{i}}\left(\frac{v_{t}}{\sigma_{k}} \frac{\partial \varepsilon}{\partial x_{i}}\right)+C_{1} S \cdot \varepsilon-C_{2} \frac{\varepsilon^{2}}{k+\sqrt{v \varepsilon}},
\end{aligned}
$$

where

$$
C_{2}=\max \left(0.43 \frac{\eta}{\eta+5}\right), \quad \eta=S \frac{k}{\eta}, \quad S=\sqrt{2 S_{i j} \cdot S_{i j}},
$$

and the constants: $\sigma_{k}$ is $1.0 ; \sigma_{\varepsilon}$ is 1.2 ; and $C_{2}$ is 1.9. In these equations, $k$ is the turbulent kinetic energy; $\varepsilon$ is the turbulent energy dissipation rate; $S$ is the mean strain rate; $v_{\varepsilon}$ is the eddy viscosity; $v$ is the fluid viscosity; $u_{j i}$ and $u_{j} u_{i}$ are the Reynolds averaged velocity in the in $i$ and $j$ direction. Hence, the turbulent flow field is determined by solving a system of four equations including the governing equations (see Eqs. (4)-(5)) and the $k-\varepsilon$ turbulence model (see Eqs. (6)-(8)) [8]. The numerical solver used in the current study is the pressure-based solver, which is well-suited for incompressible flows governed by motion based on pressure gradients. Another task compatible turbulent model is the $k-\varepsilon$ or the ReNormalized Group (RNG). In the CFD simulations, the RNG $k-\varepsilon$ turbulence model is used. RNG differs from the standard $k-\varepsilon$ in an additional sink expression in the turbulence dissipation to account for non-equilibrium strain rates and employs different values for the model coefficients [9]. The prognostic relations of turbulent kinetic energy and dissipation can be written as

$$
\begin{aligned}
& \frac{\partial k}{\partial t}+u_{j} \frac{\partial k}{\partial x_{j}}=-\bar{u}_{1} \bar{u}_{j} \frac{\partial u_{j}}{\partial x_{j}}+\frac{\partial}{\partial x_{j}}\left(\frac{K_{m}}{\sigma_{k}} \frac{\partial k}{\partial x_{j}}\right)-\varepsilon, \\
& \frac{\partial \varepsilon}{\partial t}+u_{j} \frac{\partial \varepsilon}{\partial x_{i}}=C_{\varepsilon_{1}} \frac{\varepsilon}{k} \bar{u}_{1} \bar{u}_{j} \frac{\partial u_{i}}{\partial x_{j}}+\frac{\partial}{\partial x_{j}}\left(\frac{K_{m}}{\sigma_{\varepsilon}} \frac{\partial \varepsilon}{\partial x_{j}}\right)-C_{\varepsilon_{2}} \frac{\varepsilon^{2}}{k}-R,
\end{aligned}
$$

where $k, \varepsilon, C_{\varepsilon 2}, C_{\varepsilon 2}$ are constants, [3]. Extra strain rate (Eq. 10) is given by 


$$
\begin{gathered}
R=\frac{C_{\mu} \eta^{3}\left(1-\eta / \eta_{0}\right) \varepsilon^{2}}{\left(1+\beta_{0} \eta^{3}\right) k}, \\
\eta=\frac{k}{\varepsilon}\left[\left(\frac{\partial u_{i}}{\partial x_{j}}+\frac{\partial u_{i}}{\partial x_{i}}\right) \frac{\partial u}{\partial x_{j}}\right]^{1 / 2} .
\end{gathered}
$$

The RNG $k-\varepsilon$ turbulence model got the origins in the standard $k-\varepsilon$, which where the original impetus was to improve the mixing-length model, as well as to find an alternative to algebraically prescribing turbulent length scales in moderate to high complexity flows [10].

The simulations took place after measurements in the laboratory. The main objective was to calibrate the CFD model for the different settings so that the deviations of velocities and water levels were as small as possible and determine the air entrainment at the downstream of broad-crested weir. Model geometry and boundary conditions were identical with the physical model at the same scale. The monitored points had the same position and density as the measured points as it is shown in Fig. 4.

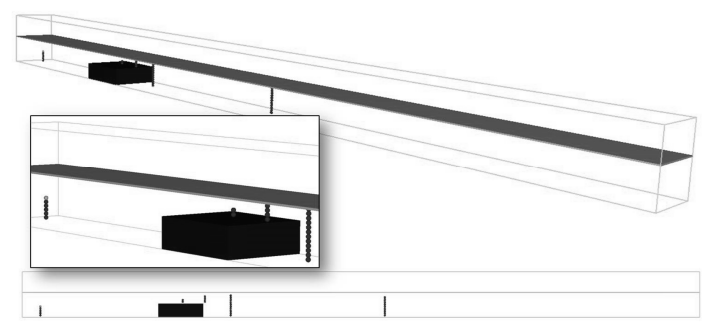

Fig. 4. Broad-crested weir (CFD model setup, with dimensions of $t=0.497 \mathrm{~m}$ and $s=0.151 \mathrm{~m}$.)

Based on previous research the mesh resolution was set to $0.00625 \mathrm{~m}$ for each simulation except the Simulation 5 where the $0.003125 \mathrm{~m}$ had to be applied for high demand of tracking algorithms of used aeration model. The most accurate turbulent model for a given problem was considered as RNG or $k-\varepsilon$ turbulent model with dynamically computed maximum turbulent mixing length.

The influence of the aeration was performed in 5 simulations with different air entraining approach to aeration modeling, with scheme as follows: without aeration (Simulation 1) where the whole problem was carried out without any air entrainment calculations, empirical aeration model (Simulation 2) based on the

$$
\frac{d V_{c a v}}{d t}=C_{a} v_{\text {production }}-C_{a} v_{\text {dissipation }},
$$




$$
\begin{aligned}
& C_{a} v_{\text {production }}=C_{e} \frac{E_{\text {turb }}}{\sigma} \rho_{l} \rho_{v} \sqrt{\frac{2}{3} \frac{P_{c a v}-P}{\rho_{l}}}\left(1-f_{\text {cav }}\right), \\
& C_{a} v_{\text {dissipation }}=C_{c} \frac{E_{\text {turb }}}{\sigma} \rho_{l}^{2} \sqrt{\frac{2}{3} \frac{P-P_{\text {cav }}}{\rho_{l}}} f_{\text {cav }},
\end{aligned}
$$

where $v_{c a v}$ is the cavitation volume fraction; $C_{a}$ is the cavitation production coefficient; $C_{e}$ is the evaporation coefficient; $C_{c}$ is the condensation coefficient; $E_{t u r b}$ is the turbulent kinetic energy; $\sigma$ is the surface tension coefficient; $P_{c a v}$ is the specified cavitation pressure; $P$ is the fluid pressure; and $f_{c a v}$ is the mass fraction of cavitation; $\rho_{l}$ and $\rho_{v}$ are the density of the liquid or vapor.

As the next approach is the aeration model with mixing and transport of the first order for water and air at $20{ }^{\circ} \mathrm{C}$ and considering surface tension (Simulation 3), a fullfledged two-phase model (Water-Air) with sharp interface and density evaluated as a function of other quantities was carried out as Simulation 4 and aeration model with an active model of tracking an adiabatic bubbles and a phase change with void initial vapor pressure of $2300 \mathrm{~Pa}$ and first order approximation to density transport equation as Simulation 5 where the equation-of-state for a bubble is the ideal gas equation,

$$
\begin{aligned}
& p=(\gamma-1) \rho C_{v}^{v a p} T, \\
& p=p_{0}\left(\frac{V_{0}}{V}\right)^{\gamma}, \\
& p^{s a t}=P V_{l} \exp \left(\frac{1 / T V_{l}-1 / T}{T V E X P}\right),
\end{aligned}
$$

where $p$ is the bubble pressure; $\rho$ is its gas density; $C_{v}{ }^{v a p}$ is the specific heat at constant volume of the vapor; and $T$ is the vapor temperature, which must be in an absolute scale. Eq. (16) is used, if there are no sources of mass or energy for a bubble and the phenomena will behave as though it is adiabatic, changing pressure from some initial state $\left(p_{0}, V_{0}\right)$ to a new state $(p, V)$. When phase changes occur it is necessary to have an analytical relation that expresses the saturation pressure of a vapor in terms of its temperature. The proper relation is described in Eq. (17). The rate of phase change is typically modeled as proportional to something that measures the deviation from saturation conditions [11].

$$
\text { Net mass transfer }=\sqrt{\frac{M}{2 \pi R}}\left(a_{v a p} \frac{p_{l}^{\text {sat }}}{\sqrt{T}}-a_{c o n} \frac{\rho_{v}}{\sqrt{T_{v}}}\right)
$$


where $M$ is the molecular weight of the vapor; $R$ is the vapor gas constant; $T$ is temperature; and subscripts $l$ and $v$ refer to liquid and vapor states. The superscript sat on $p_{l}$ indicates the saturation pressure corresponding to liquid temperature $T_{v}$. Finally, the coefficients $a_{v a p}$ and $a_{c o n}$ are the accommodation coefficients for evaporation and condensation, respectively [12].

\section{Evaluation of the model}

The measured velocity fields were set as verification parameters for CFD numerical model evaluation (Table I). Previous research showed that the mesh resolution and turbulent model mentioned above are proper.

Table I

Comparison of the velocity fields of the physical experiment and the simulation 1 as a verifying benchmark simulation

\begin{tabular}{|c|c|c|c|c|c|c|c|c|c|}
\hline . & $\begin{array}{l}0 \\
0 \\
0 \\
0\end{array}$ & 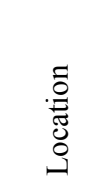 & 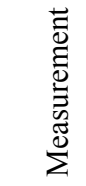 & 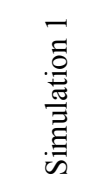 & $\begin{array}{l}.0 \\
\stackrel{0}{0} \\
\stackrel{0}{0}\end{array}$ & $\begin{array}{l}0 \\
0 \\
0 \\
0\end{array}$ & 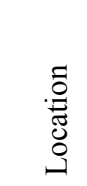 & 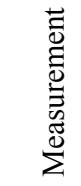 & 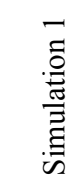 \\
\hline \multirow{11}{*}{1} & 1 & 0.030 & 0.243 & 0.209 & \multirow{12}{*}{2} & 1 & 0.020 & 0.217 & 0.202 \\
\hline & 2 & 0.050 & 0.243 & 0.215 & & 2 & 0.040 & 0.232 & 0.208 \\
\hline & 3 & 0.080 & 0.242 & 0.220 & & 3 & 0.060 & 0.241 & 0.215 \\
\hline & 4 & 0.100 & 0.239 & 0.225 & & 4 & 0.080 & 0.244 & 0.222 \\
\hline & 5 & 0.120 & 0.240 & 0.229 & & 5 & 0.100 & 0.248 & 0.229 \\
\hline & 6 & 0.140 & 0.241 & 0.233 & & 6 & 0.120 & 0.248 & 0.235 \\
\hline & 7 & 0.160 & 0.238 & 0.238 & & 7 & 0.140 & 0.251 & 0.241 \\
\hline & 8 & 0.180 & 0.237 & 0.242 & & 8 & 0.160 & 0.253 & 0.247 \\
\hline & 9 & 0.200 & 0.237 & 0.245 & & 9 & 0.180 & 0.249 & 0.251 \\
\hline & 10 & 0.220 & 0.239 & 0.246 & & 10 & 0.200 & 0.255 & 0.253 \\
\hline & 11 & 0.240 & 0.237 & 0.247 & & 11 & 0.220 & 0.252 & 0.253 \\
\hline \multirow{4}{*}{3} & 1 & 0.170 & 0.429 & 0.547 & & 12 & 0.240 & 0.245 & 0.250 \\
\hline & 2 & 0.190 & 0.510 & 0.534 & \multirow{2}{*}{4} & 1 & 0.170 & 0.985 & 0.895 \\
\hline & 3 & 0.210 & 0.504 & 0.515 & & 2 & 0.190 & 1.025 & 0.962 \\
\hline & 4 & 0.230 & 0.503 & 0.495 & & & & & \\
\hline
\end{tabular}

The overall deviation between the Simulation 1 and measurement is in average $5.45 \%$ and in median $3.96 \%$. The biggest discrepancy is located at the $1.9 \mathrm{~cm}$ above the front edge of the crest, which represents the deviation of $27.66 \%$ (Fig. 5) but considering the character of the flow at the location and the measurement methodology after $5 \%$ exclusion of the best and the worst results the overall average deviation decreases at $4.70 \%$ and $3.96 \%$ at the median value. 

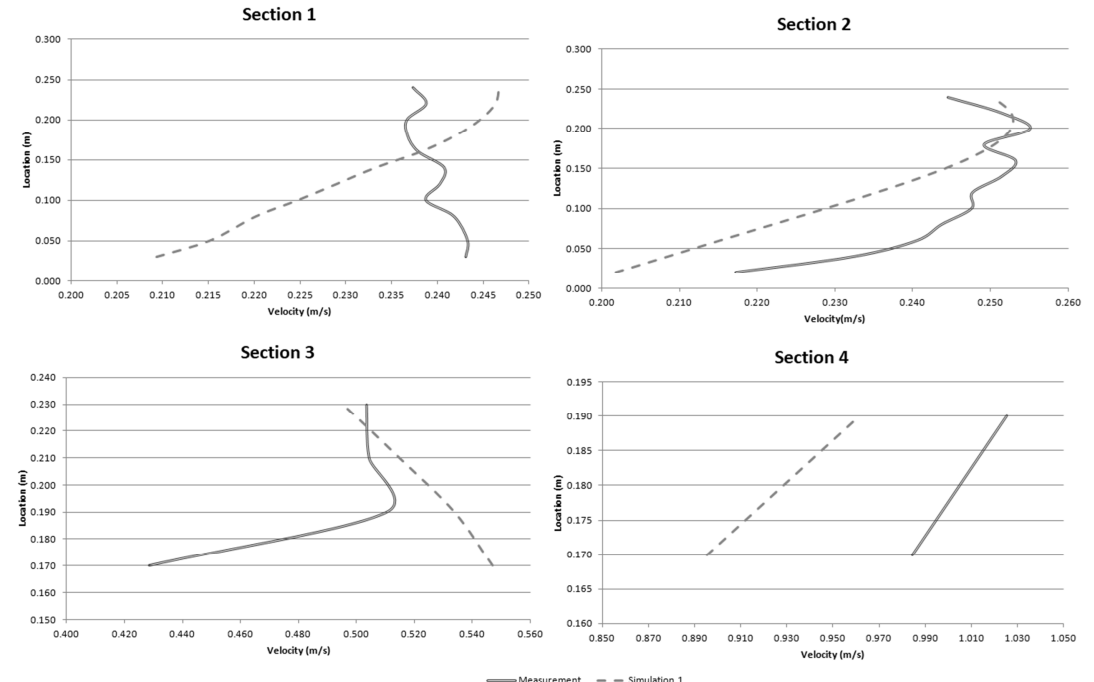

Fig. 5. Comparison of the velocity fields of Simulation 1 and the measurement

\section{Results and discussion}

The design of the weir crest, water level regime coupled with boundary conditions is creating conditions for highly turbulent, aerated flow downstream of the structure. The presented complicated behavior of the fluid flow is hard to track even on the scaled models in laboratory. Performed simulations are resulting to high computer demands together with long computation times where selection of approach to the air entrainment phenomena is very important.

Simulation 1 was set as benchmark simulation for four different alternatives of air entrainment along with detailed matching analysis of velocity fields and free surface elevation. Simulation 2 with its empirical Aeration model based on Eq. (13), (14), (15) versus benchmark simulation resulted to average deviation of $0.36 \%$ on the velocity parameter. Simulation 3 as the Aeration model with mixing and transport of the first order versus benchmark simulation resulted to average deviation of $1.12 \%$ on the velocity parameter. Full-fledged two-phase model (Water-Air) labeled as Simulation 4 versus benchmark simulation resulted to average deviation of $36.03 \%$ on the velocity parameter. Simulation 5 with its Aeration model with an active model of tracking an adiabatic bubbles based on the Eq. (16), (17), (18) versus benchmark simulation resulted to average deviation of $7.26 \%$ on the velocity parameter (Fig. 6).

Simulation 2 versus benchmark simulation resulted to average deviation of $0.82 \%$ deviation on the free surface elevation. Simulation 3 versus benchmark simulation resulted to average deviation $1.53 \%$ deviation on the free surface elevation. Simulation 4 versus benchmark simulation resulted to average deviation of $8.34 \%$ deviation on the free surface elevation. Simulation 5 versus benchmark simulation resulted to average deviation $25.99 \%$ deviation on the free surface elevation (Fig. 7). 

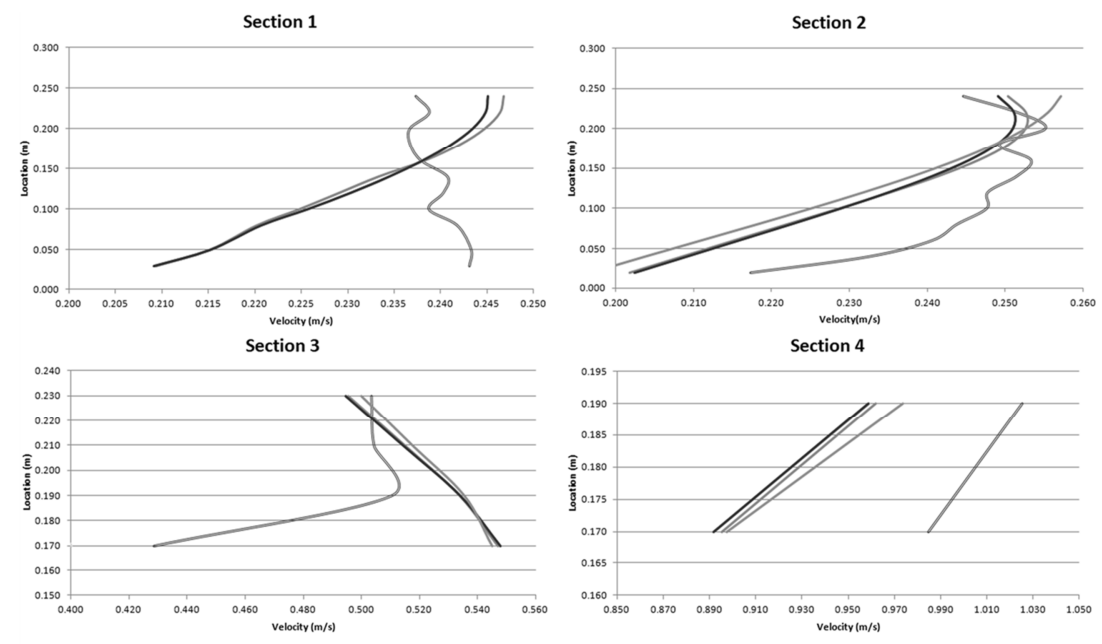

Fig. 6. Velocity fields for different approaches of air entrainment modeling

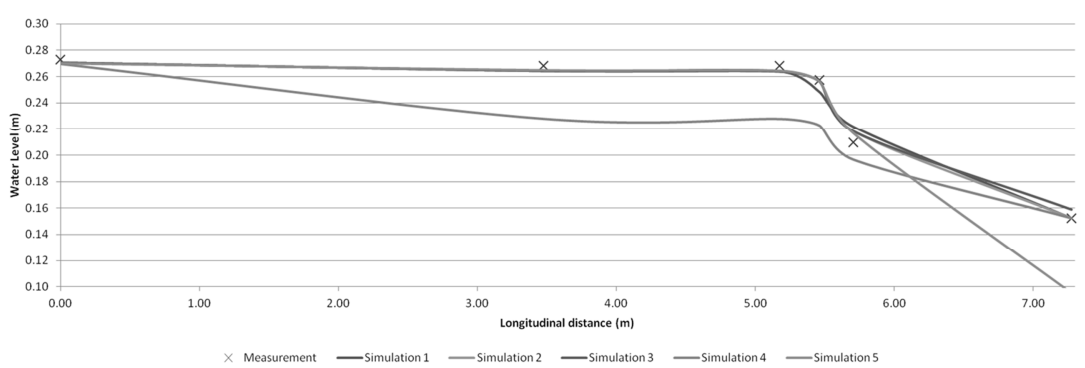

Fig. 7. Water level for different approaches of air entrainment modeling

Hydraulic jumps are often problematic when it comes to numerical modeling and proper approach to phenomena from simulation point of view. From sectional 2D CFD models (Fig. 8) through symmetric 3D models to fully developed 3D approach with turbulence consideration and aeration The most complex problems need modeling to take place in realistic three-dimensional enclosures, which makes it essential to find a compromise between the accuracy of the models and their CPU efficiency [13].

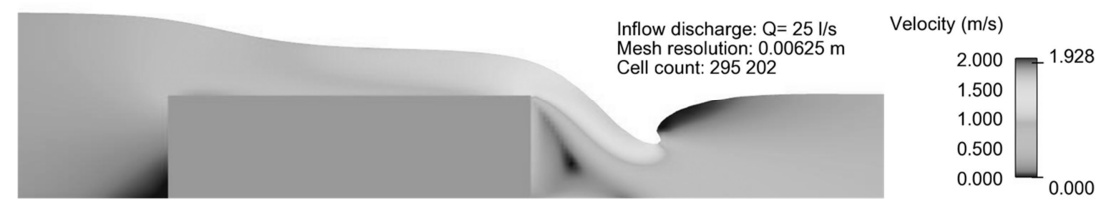

Fig. 8. Air entrainment and free surface modeling of fully turbulent flow near the broad-crested weir 
Besides the deviations of water level and velocity it has to be point out that the empirical model should be considered as the optimal also in terms of computation demands (Fig. 9).

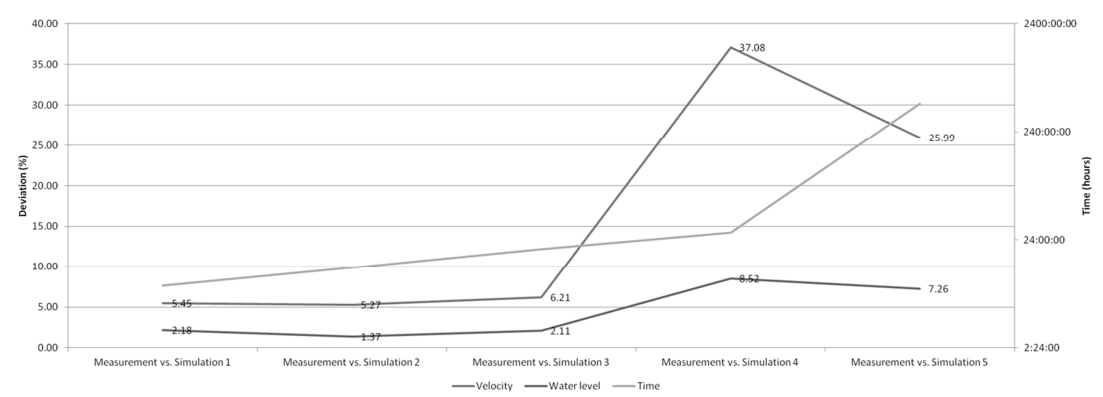

Fig. 9. Accuracy and time consumption of different approaches to air entrainment modeling

According to the previous research, the air entrainment can generate high residence time due to dead-zones in fluid flow which can appear in many others engineering applications. This approach is based on the velocity gradient tensor of fluid flow, which is pre-calculated with the tool of computational fluid dynamics [14].

\section{Conclusion}

The results are based on the research at the Department of Hydraulic Engineering, Faculty of Civil Engineering Slovak University of Technology in Bratislava with close cooperation with Institute of Water Structures, Faculty of Civil Engineering, Brno University of Technology. One of the important goals was determination of the most accurate approach related to the CFD modeling of the air-water interaction. A literature review concerning air entrainment interactions indicates that only a small number of publications deal with air entrainment as to its quantification. Considering the results for free surface flow tracking the most accurate model for a research is the empirical Aeration model referred to Simulation 2 with absolute deviations to physical model measurements of $1.37 \%$. In terms of velocity evaluation is the Empirical model the most effective as well with total deviation of $5.27 \%$.

\section{Acknowledgements}

This article was created with the support of the Grant Agency VEGA, number 1/0361/17. This article was created with the support of the Grant Agency VEGA, number $1 / 0800 / 17$.

This article was created with the support of the Grant Agency KEGA, number 053STU-4/2017. 
This article was created with the support of the Grant of the Slovak Technical University in Bratislava for support the excellent teams of young researchers, HYMODYK.2016.

The contribution was developed within the frame and based on the financial support of the APVV-15-0489 project 'Analysis of droughts by multi-criterial statistical methods and data mining from the viewpoint of prevention structures in a landscape'.

\section{References}

[1] Ǩ́ha J., Zachoval Z. Flow characteristics at trapezoidal broad-crested side weir, J. Hydrol. Hydromech, Vol. 63, No. 2, 2015, pp. 164-171.

[2] Mäsiar E., Kamenský J. Hydraulika II, Textbook (in Slovak) Slovak University of Technology in Bratislava, 1989.

[3] Picek T., Havlík A., Mattas D., Pressure flow and overflow bridges, J. Hydrol. Hydromech, Vol. 52, No. 3, 2004, pp. 185-192.

[4] Guo Z. W., Chen F., Wu P. F., Qian Z. D. Three-dimensional simulation of air entrainment in a sump pump, J. Hydaulic Eng. Vol. 143, No. 9, 2017, pp. 1-9.

[5] Souders D. T., Hirt C. W. Modeling entrainment of air at turbulent free surfaces, World Water and Environmental Resource Congress, Salt Lake City, Utah, US, 27 June-1 July 2004, pp. 1-9.

[6] Chanson H., Murzyn F. Froude similitude and scale effects affecting air entrainment in hydraulic jumps, World Environmental and Water Resources Congress, Honolulu, Hawaii, United States, 12-16 May 2008, Paper 240.

[7] Emiroglu E. M. Estimating flow characteristics of different weir types and optimum dimensions of downstream receiving pool, J. Hydrol. Hydromech, Vol. 58, No. 4, 2010, pp. 254-260.

[8] Shih T. H., Liou W. W., Shabbir A., Yang Z., Zhu J. A new k- $\varepsilon$ eddy-viscosity model for high Reynolds number turbulent flows-model development and validation, Computers and Fluids, Vol. 24, No. 3, 1995, pp. 227-238.

[9] Yakhot V., Orszag S. A., Thangam S., Gatski T. B., Speziale C. G. Development of turbulence models for shear flows by a double expansion technique, Physics of Fluids A: Fluid Dynamics, Vol. 4, No. 7, 1992, pp. 1510-1520.

[10] Tutar M., Oguz G. Large eddy simulation of wind flow around parallel buildings with varying configurations, Fluid Dynamics Research, Vol. 31, No. 5-6, 2002, pp. 289-315.

[11] Möller G., Detert M., Boes R. M. Vortex-induced air entrainment rates at intakes, J. Hydraulic Engineeing, Vol. 141, No. 3, 2016, Paper 04015026.

[12] Kim J. J., Baik J. J. A numerical study of the effects of ambient wind direction on flow and dispersion in urban street canyons using the RNG $k-\varepsilon$ turbulence model, Atmospheric Environment, Vol. 38, No. 19, 2004, pp. 3039-3048.

[13] Sazhin S. Modeling of sprays using computational fluid dynamics codes, Pollack Periodica, Vol. 4, No. 1, 2009, pp. 5-16.

[14] Karches T. Detection of dead-zones with analysis of flow pattern in open channel flows, Pollack Periodica, Vol. 7, No. 2, 2012, pp. 139-146. 